DOI: 10.20472/IAC.2019.048.002

\author{
HAYA AL-AJLANI \\ University of Ghent, Belgium
}

\title{
UNDERSTANDING STATE FRAGILITY
}

\begin{abstract}
:
The fragility of a state is often deemed an impediment to prosperity and sustainable development. A fragile government is unable to realize its full potential, jeopardizing the opportunities available for citizens to improve their quality of life. This notion of "arrested development" is echoed in the recent OECD (2018) and World Bank reports (2016), where fragility was identified as a main encumber to achieving the 2030 agenda for Sustainable Development Goals (SDGs). Yet, existing literature remains inconclusive with regard to defining and measuring state fragility. Existing definitions vary across literature, and existing indices comprise a variety of indicators measuring not only state fragility but also other factors that would strain the performance of any well-functioning government. Consequently, such indices widen the breach between the theoretical definitions of state fragility and the empirical analysis. In this paper, we aim to provide a concrete definition of state fragility, and an index that is closely related to our definition. Building on the notion of statehood, we define fragile states as governments that lack legitimacy such that they fail to deliver basic services, and their authority is not endorsed by citizens. Therefore, our index of state fragility measure two elements: the provision of key services and the political legitimacy of the government (i.e. the endorsement the state receives from citizens). In our measurement of these two components, we use data envelopment analysis to condition on the level of economic development of the country. This conditionality helps differentiate between fragility issues, and development issues; is the poor provision of basic services or weak political legitimacy due to limited resources, or due to a fragile government? Our results show that

Iraq, Nigeria, Yemen, Saudi Arabia, and Pakistan are the most fragile countries, respectively. In fact, compared to countries with a similar GDP per capita level, Saudi Arabia performs poorly with respect to all aspects of political legitimacy. Our results also suggest that fragility is a vicious cycle; states deemed fragile in a given year are likely to be deemed fragile in the years to follow. The index suggested in this paper can be a guiding tool for governments to evaluate their relative performance and identify areas that require improvements, in hopes of providing a better quality of life for citizens.
\end{abstract}

\section{Keywords:}

State fragility, legitimacy, Data envelopment analysis 